$$
\text { PPPL- - 3212 }
$$

\title{
SELF-SIMILARITY AND TRANSPORT \\ IN THE STANDARD MAP
}

\author{
S. Benkadda ${ }^{a}$, S. Kassibrakis ${ }^{a}$ and R. B. White \\ Plasma Physics Laboratory, Princeton University, P.O.Box 451, \\ Princeton, New Jersey 08543 \\ G. M. Zaslavsky
}

Courant Institute of Mathematical Sciences, New York University, 251 Mercer Street, New York, New York 10012, and Physics Department, New York University, \& Washington Place, New York, New York 10003

\begin{abstract}
Anomalous transport is investigated for the Standard Map. A chain of exact self similar islands in the vicinity of the period 5 accelerator island is found for a particular value of the map parameter. The transport is found to be superdiffusive with an anomalous exponent related to the characteristic temporal and spatial scaling parameters of the island chain. The value of the transport exponent is compared to the theory. The escape time distribution and Poincaré recurrence distribution are found to have power-like tails and the corresponding exponents are obtained and compared to the theory.
\end{abstract}

PACS numbers: $05.45 .+b, 47.52 .+\mathrm{j}, 05.60 .+\mathrm{w}, 47.53 .+\mathrm{n}$

a) Permanent address: Equipe Turbulence Plasma, URA 773, CNRS-Université de Provence. Institut 


\section{DISCLAIMER}

This report was prepared as an account of work sponsored by an agency of the United States Government. Neither the United States Government nor any agency thereof, nor any of their employees, make any warranty, express or implied, or assumes any legal liability or responsibility for the accuracy, completeness, or usefulness of any information, apparatus, product, or process disclosed, or represents that its use would not infringe privately owned rights. Reference herein to any specific commercial product, process, or service by trade name, trademark, manufacturer, or otherwise does not necessarily constitute or imply its endorsement, recommendation, or favoring by the United States Government or any agency thereof. The views and opinions of authors expressed herein do not necessarily state or reflect those of the United States Government or any agency thereof. 


\section{DISCLAIMER}

Portions of this document may be illegible in electronic image products. Images are produced from the best available original document. 
Méditerranéen de Technologie, F-13451 Marseille Cedex 20, France 


\section{INTRODUCTION}

The standard map ${ }^{1}$ is one of the most frequently occurring models in many different applications. Written in the form

$$
p_{n+1}=p_{n}-K \sin x_{n}, \quad x_{n+1}=x_{n}+p_{n+1}
$$

it has become a paradigm for the study of properties of chaotic dynamics in Hamiltonian systems. In spite of its apparent simplicity, unexpected difficulties in attempts to write down a kinetic model for Eq. 1.1, brought researchers back to the problems of characterizing chaos per se and describing chaotic trajectories. More specificially, in studying the occurrence of chaos one has to deal with local properties of the trajectory dynamics (local instability, Lyapunov exponents, bifurcations, etc.). At the same time the typical object of research is to find large scale properties of the trajectories and characterize the kinetics and the evolution of moments in large time asymptotics.

The first surprise was the numerical discovery ${ }^{1}$ and the corresponding theory ${ }^{2-6}$ that the diffusion constant $\mathcal{D}$ is a quasi-oscillating function of the parameter $K$ in Eq. 1.1 for $K>1$. The corrections are of order one and remain significant up to values of $K \sim 40$. The oscillations are due to long time correlations which persist well above chaotic threshold, but the motion is diffusive, and can be described by a diffusion constant $\mathcal{D}$, with oscillatory dependence on $\mathrm{K}$ given by the theory.

A more fundamental difference between "perfectly chaotic" systems, like Sinai's billard, and more realistic systems, like the standard map, is the existence of islands, making phase space a complicated mixture of small nonchaotic domains and stochastic regions. The presence of the islands implies much stronger deviations in long time asymptotics than can be 
described by simply a change in the diffusion constant. The existence of anomalous transport of a nondiffusional character for values of $K$ taken in the vicinity of a set $\left\{K_{c}\right\}$ which includes $K \sim 1$ and accelerator mode values has been previously conjectured ${ }^{7-11}$. A new understanding of the anomalous transport for the standard map was proposed where the connection to the so-called Lévy flights process was established ${ }^{12,13}$. It was previously shown that the islands have a strong effect if the parameter $K$ is taken near some critical values such as $K \sim 1$ (transition to global chaos ${ }^{8,2,14}$ ), the large peaks near $K_{n}=2 \pi n$ (accelerator modes ${ }^{15}$ ), and some other fractional accelerator modes ${ }^{7,9,10}$. In this article, the connection will be "visualized" in an explicit and quantitative way.

The anomalous transport for the model given by Eq. 1.1 can be expressed in general as superdiffusion described by the second moment of $p$,

$$
\left\langle p^{2}\right\rangle=c \cdot t^{\mu}, \quad(t \rightarrow \infty)
$$

where

$$
1<\mu<2
$$

in contrast to normal transport (diffusion) with $\mu=1$. In fact the expression in Eq 1.2 is the global result of some deeper properties of anomalous transport, characterized by specific Lévy-type processes ${ }^{16}$ which occur in numerous physical applications (see for example a previous review ${ }^{17}$ where such processes were named "strange kinetics").

The origin of anomalous transport has been connected to the local topological properties of the phase space domain near islands (the boundary layer) ${ }^{18}$ and a self-similar structure of the domain ${ }^{19}$. A quantitative theory has been proposed using a fractional generalization of the diffusion (Fokker-Planck-Kolmogorov) equation ${ }^{20,21}$. The renormalization group was 
used to obtain an explicit expression for the transport exponent $\mu$ in Eq. 1.2 as a function of space-time scaling constants for islands in the boundary layer. For some values of the map parameter there is a self-similar hierarchy of sub-islands in the boundary layers. For these values a theoretical expression for $\mu$

$$
\mu=\ln \lambda_{S} / \ln \lambda_{T}
$$

can be derived, where $\lambda_{S}$ is a scaling parameter for the island area and $\lambda_{T}$ is a scaling parameter for the rotation frequency of orbits within the island ${ }^{20,21}$. Recently these results were confirmed for the web-map

$$
u_{n+1}=v_{n}, \quad v_{n+1}=-u_{n}-K \sin v_{n}
$$

using simulations requiring very exact numerical methods ${ }^{22,23}$.

In this article we follow the same procedure to find the transport exponent $\mu$ for the standard map. There is a significant difference between the standard map and the web map. While the flights in the web map are of spiral type and symmetric in the $u-$ or $v-$ directions in phase space, they are of a parabolic and nonsymmetric type for the standard map. For an accelerator mode a flight along $p$ means that $p \sim t$ and thus $x \sim t^{2}$. This rapid increase of $x$ leads to a dangerous accumulation of the roundoff errors for the phase variable $x$, and makes computational difficulties in determining the anomalous transport exponent $\mu$ and comparing it with the theoretical prediction Eq. 1.4. In fact the absence of a symmetry leads to a corresponding modification of the result of Eq. 1.4 and this generalization will be given below.

In this work we present a number of results for the standard map. In section II we give an example of a self-similar hierarchy of islands in the boundary layer. This example is used to 
display results which we believe have a very general character. In section III we describe the numerical techniques used to investigate long time asymptotic behavior. In section IV the scaling properties of the hierarchy including the island areas, rotation frequencies, escape time distributions, and the statistical properties of trajectories are discussed. In section $\mathrm{V}$ the renormalization of the escape time according to the generation of islands in the heirarchy is examined. We show how all these properties are related to the transport exponent $\mu$ in Eq. 1.2 and how the anomalous transport occurs. Our results confirm the previously discovered property of chaotic trajectories to be not "sufficiently chaotic"18-21, i.e. to have long lasting

memory for the major part of the trajectory ${ }^{9,10}$ unless the value of the control parameter $K$ is taken in specific locunas (or even at a point) where the islands collapse ${ }^{23}$. We also find the anomalous exponent to be related to the characteristic temporal and spatial scaling parameters of the island chain. Finally in section VI we give some concluding remarks.

\section{SELF-SIMILAR HIERARCHY OF ISLANDS IN THE BOUNDARY LAYER}

The existence of a fine structure of the island patterns in phase space and its properties are fundamental in the study of anomalous transport. Indeed the existence of such islands and their boundaries alters the pattern of diffusion dynamics. Because of the PoincaréBirkhoff theorem ${ }^{4}$, in the neighborhood of a generic periodic orbit there are satellite elliptic orbits of smaller sizes, each of which in turn has satellite elliptic orbits, and so forth. These elliptic orbits are ordered forming a chain of islands around islands. For particular values of $\mathrm{K}$ the island chain can assume a self similar character. In order to make explicit this self-similarity of islands we found values of the perturbation parameter $\mathrm{K}$ for which there is a self-similar hierarchy of subislands in the boundary layer. In Fig. 1 is shown four 
generations of islands related to the accelerator mode of period 5 . The generation 0 island is the accelerator island of period 5, top right, and subsequent generations are seen by proceeding counterclockwise. The small rectangle in each plot shows the domain which is blown up in the next generation. We will refer to this chain as a boundary island chain (BIC). Small changes in the perturbation parameter produces different chains, with varying numbers of islands in each generation. To obtain this example we searched in the vicinity of the critical perturbation parameter value related to this accelerator mode, until we found 3 generations of BIC's of 11 islands each for $K_{c}=6.476939$. This set of island chains is the first such self-similar BIC hierarchy found for the standard map. Another example of a BIC hierarchy with 8 islands will be published soon ${ }^{23}$. The Poincare plots were obtained by initiating a point near an X-point and advancing many steps. The island boundary is much darker than the surrounding domain because of the long time necessary for the orbit to escape from it. In fact for the higher generation islands the orbit takes a very long time to escape. This stickiness occurs at any generation of the BIC. The number of generations can be increased further by adjusting the value of $K_{c}$ through the addition of more digits.

In this paper, we wish to analyse more closely the effect this BIC self-similarity has on a chaotic orbit. In other words we want to determine the stickiness of the island. Because we concentrate only on the behavior near the island, our approach is mainly a local one.

There are typically two quantities for characterizing a BIC. One is related to the area of islands and the other is related to a characteristic time scale of the islands which can be for instance the winding frequency of an orbit about the island O-point. 


\section{NUMERICAL CONSTRUCTION OF STICKY DOMAINS}

In order to investigate the spatio-temporal properties related to self-similarity we need to determine accurately the phase space domains embedding the accelerator modes and susceptible to contain the BIC's. Long time trapping is only caused by such domains, and for numerical reasons the domain of initial conditions used should correspond to them. To make rapid numerical calculations of trapping time or exit time for a given set, we divide that part of phase space of interest into $N \times N$ domains with a rectangular grid with boundaries $x_{1}, x_{1}+\Delta x$ and $p_{1}, p_{1}+\Delta p$. The grid provides an immediate coarse graining of phase space $x, p$ into $i, j$ with

$$
\begin{aligned}
& j=\left(x-x_{1}\right) * N / \Delta x+1 \\
& i=\left(p-p_{1}\right) * N / \Delta p+1
\end{aligned}
$$

To construct domains for the study of long time trapping, we have developed a suitable numerical procedure ${ }^{24,14}$. This procedure consists of initiating a point in the stochastic sea, applying the map a large number of times, allocating the value 1 to all non visited cells and the value 0 to the visited ones, thus constructing a matrix $n(i, j)=0$ in the stochastic sea, and $n(i, j)=1$ otherwise. This matrix simply defines the coarse grain evaluation of the extent of the stochastic sea and the islands which are distinguishable with the coarse graining used. It depends only on the value of the perturbation parameter $K$ and the mesh location and size. Clearly the number of visits to the domain covered by the mesh must be much larger than $N \times N$, otherwise there will remain elements $n(i, j)$ equal to 1 which should be 0 . We then sort the matrix $n(i, j)$ according to each distinct island domain, with $n(i, j)=k$ within island $k$, zero otherwise, and measure the area of each island. There 
results a list of all islands, sorted according to area. We select afterwards an island for study, and construct a boundary set B of this island, consisting of all $i, j$ in the stochastic sea, but within some distance $d_{0}$ of the island. Define a matrix $b(i, j)=1$ within B and zero otherwise. The set B quantifies the concept of being close to the island in question.

Rapid examination of trapping times, escape times, and recurrence times is now possible with the matrix $b(i, j)$. Advance a point initiated in the stochastic sea according to the map. At each step $t$ evaluate $b(t)=b(i, j)$. Then note that $b(t)-b(t-1)=1$ if the point has just entered $B,-1$ if it has just left, and is zero otherwise. Each such instance is recorded, giving a data set consisting of the distribution of trapping times and recurrence times for the domain $\mathrm{B}$. This procedure eliminates the necessity of evaluating any metric quantities (such as distance from an island) during the simulation.

We measure orbit averaged trapping times and recurrence times by following a long orbit initiated outside the domain B in the stochastic sea. Escape time, calculated by initiating many orbits inside the domain B and measuring first exit, biases the result according to the density assumed inside B, and cannot a priori be expected to be equal to the orbit averaged value. We have used uniform initial density to define escape times.

We also used a second method for constructing sticky domains. It consists simply in initiating a point near an island boundary, typically near an X-point, and letting it evolve under the mapping sufficiently long to generate the set $\mathrm{B}$, but not long enough for it to escape far into the stochastic sea. If necessary, $\mathrm{B}$ can be then enlarged by adding to it all mesh points within some range $\Delta i, \Delta j$.

The long time tail in the distribution of escape times and recurrence times is independent of the domain B provided only that B contains the island boundary domain. It is numerically 
convenient that this set be as small as is consistent with this requirement, to optimize computing efficiency, and to be certain that many data points in the sticky domain near the island boundaries are present to obtain good statistics for the long time tails.

\section{SPATIO-TEMPORAL SELF-SIMILARITY OF THE ACCELERATOR MODE}

\section{ISLANDS}

The self-similar property of the BIC's areas can be expressed in the form

$$
\Delta S_{k-1}=\lambda_{S} \Delta S_{k}, \quad \lambda_{S}>1
$$

where $k$ is the order of generation of the BIC, and $\Delta S_{k}$ is the area of all islands in the $k$-th generation of the BIC. Numerically we compute these areas by counting the cells belonging to an island after having 'squared" the domain using the method described above. Then, the elementary area of one cell is $\Delta p \Delta x / N^{2}$.

To display the temporal self-similarity, we use the winding number which is a characteristic frequency of the islands. Let us denote it as $\omega_{k}$ and compute it in the interior of the island. It is indeed the rotation frequency of a stable orbit $\omega=1 / \Delta t$ with $\Delta t$ the time interval (steps) required to completely circle the island O-point. It is computed as a function of the distance from the center of the island so that it provides the profile ${ }^{25} \omega(x)$. We determine the center of the island by analyzing the Poincaré sections. For the simulation corresponding to the BIC displayed in Fig 1, we typically let the orbit make 50 rotations and used $10^{3}$ initial conditions for computing the internal rotation frequency. The initial condi- 
tions are uniformly distributed on a segment linking the center of the island to its external boundary. This assures us good precision in our computation. Figure 2 shows the rotation frequency profiles for the four generations of the BIC shown in Fig. 1. The determination of $\omega$ becomes very noisy near the island edge as the separatrix is approached. We use the value at the O-point, $\omega_{k}(0)$ to characterize the time scale of island $\mathrm{k}$. The self-similar property of the winding numbers of the islands can be written as

$$
\omega_{k-1}=\lambda_{T} \omega_{k}, \quad \lambda_{T}>1
$$

The scaling parameters $\lambda_{S}$ and $\lambda_{T}$ are respectively the characteristic parameters of the spatial and temporal self-similarity of the chain of islands under consideration.

To illustrate the existence of the scaling related to the spatio-temporal self-similarities given by Eqs. 4.1,4.2, let us consider the set of subislands which belong to the main island of the accelerator mode of period 5 displayed in Fig.1. Variables related to these islands will be labeled " 0 " while for the next generations of islands we use "1,2,...", as shown in Fig. 1. Table 1 shows the island-set properties. The second column gives the number of islands in the generation of order $\mathrm{k}$. The exponential dependence on $k$ of the island area and winding frequency is clearly seen.

\section{TABLE 1}

\begin{tabular}{|l|l|l|l|l|l|c|}
\hline$k$ & $n_{k}$ & $\omega_{k}(0)$ & $\lambda_{T}$ & $\delta S_{k}$ & $\Delta S_{k}$ & $\lambda_{S}$ \\
\hline 0 & 5 & $2.34 \times 10^{-2}$ & - & $6.58 \times 10^{-3}$ & $3.29 \times 10^{-2}$ & - \\
1 & $5 \times 11$ & $2.39 \times 10^{-3}$ & $\mathbf{9 . 7 9 1}$ & $3.12 \times 10^{-5}$ & $1.72 \times 10^{-3}$ & $\mathbf{1 9 . 1 6}$ \\
2 & $5 \times 11 \times 11$ & $2.26 \times 10^{-4}$ & $\mathbf{1 0 . 5 8}$ & $7.47 \times 10^{-8}$ & $4.52 \times 10^{-5}$ & $\mathbf{3 8 . 0 2}$ \\
3 & $5 \times 11 \times 11 \times 11$ & $2.18 \times 10^{-5}$ & $\mathbf{1 0 . 3 6}$ & $1.81 \times 10^{-10}$ & $1.20 \times 10^{-6}$ & $\mathbf{3 7 . 5 9}$ \\
\hline
\end{tabular}


The total area of all islands at generation $\mathrm{k}$ is

$$
\Delta S_{k}=n_{k} \delta S_{k}
$$

where $\delta S_{k}$ is the area of a single island of order $\mathrm{k}$. When both $\mathrm{k}, \mathrm{k}-1$ refer to island sets containing 11 islands $(\mathrm{k}=2,3)$ the scaling parameters characterizing the self-similarity are approximately constant and given by:

$$
\lambda_{S}=37.8 \pm 0.7, \quad \lambda_{T}=10.5 \pm 0.2
$$

There is another kind of self-similarity of the system, which is related to the moments of the probability distribution function. Indeed there are two ways to consider the particle transport in the standard map: on the cylinder $(x \bmod 2 \pi)$ or in the infinite phase space in $x$ and $p$. Let us mention that in numerous publications on transport in the standard map, the moments concern only transport in the $p$ direction. However, this map being asymmetric it is of interest to consider both directions $x$ and $p$ in transport computations. Indeed in some physical problems one needs to know the phase evolution, which can be derived only in the latter case. Actually both representations were considered in this study. Before discussing the results of the simulations, let us emphasize the differences between these representations. For that we shall consider the transport near the accelerator mode in a BIC exactly self-similar with a number of islands equal to the main resonance chain.

On the cylinder, for the normal diffusion case $\left\langle p^{2}\right\rangle \sim t$ as does $\left\langle x^{2}+p^{2}\right\rangle$. The angular brackets denote averaging over a large number of initial conditions. For the accelerator mode both $\left\langle p^{2}\right\rangle$ and $\left\langle x^{2}+p^{2}\right\rangle$ scale as $t^{2}$. We can expect that in a general case of self-similar transport for any BIC, the value of the exponent of the anomalous diffusion $\mu$ should be 
bounded by the exponents relative to these two cases, that is $1<\mu<2$.

In infinite phase space, these moments do not scale in the same manner. Indeed, for normal diffusion $\left\langle p^{2}\right\rangle \sim t$ while $\left\langle x^{2}+p^{2}\right\rangle \sim t^{3}$ and for the accelerator mode chain $\left\langle p^{2}\right\rangle \sim t^{2}$ while $\left\langle x^{2}+p^{2}\right\rangle \sim t^{4}$ so that for a general case of anomalous transport in the plane we obtain $3<\mu<4$. Define $\mu_{x}$ and $\mu_{p}$ by the expressions

$$
\begin{gathered}
\left\langle p^{2}\right\rangle=c_{1} \times t^{\mu_{p}}, \quad x(\bmod 2 \pi) \\
\left\langle x^{2}+p^{2}\right\rangle=c_{2} \times t^{\mu_{x}}, \quad(t \rightarrow \infty)
\end{gathered}
$$

Fig. 3 shows these moments for computations done in the case of the island chain of Fig. 1, i.e, $K=K_{c}=6.476939$. From the map definition Eq 1.1 one can see that the change of $x$ per step is $\Delta x=p(t)$, and this, together with Eq. 4.5 for $\left\langle p^{2}\right\rangle$, gives

$$
\left\langle x^{2}+p^{2}\right\rangle \sim\left\langle x^{2}\right\rangle \sim t^{\mu_{p}+2}, \quad(t \rightarrow \infty)
$$

and

$$
\mu_{x}=\mu_{p}+2 .
$$

This relation is indeed confirmed, within the errors of slope determination, by the computations of the moments, plotted in Fig. 3, which provides the values

$$
\mu_{p}=1.42 \pm 0.15, \quad \mu_{x}=3.66 \pm 0.1
$$

The analytic expression given by Eq. 1.4 gives $\mu=1.54 \pm .02$ which is in good agreement with the value of $\mu_{p}$ obtained from Fig. 3 .

Similar results, giving good agreement between the predicted value of the anomalous diffusion exponent Eq. 1.4 and its directly computed value have been obtained also for the 
web map ${ }^{22}$. However, due to the symmetry of the web map, $\mu$ has to be compared to $\mu_{x}$ while for the standard map it is compared to $\mu_{p}$. Another value of $K_{c}$ in the standard map corresponding to an 8 th-order resonance set was considered ${ }^{23}$ giving also good agreement between Eqs. 1.4 and 4.8 .

We have also considered higher moments of the probability distribution function. For large time asymptotics of these moments $\left\langle R^{2 m}\right\rangle$ with $R^{2}=x^{2}+p^{2}$, it is shown from the simulations that

$$
M^{2 m} \equiv\left\langle R^{2 m}\right\rangle \sim C \times t^{\mu_{m}}, \quad(t \rightarrow \infty)
$$

Figs. 4 and 5 show respectively these moments for the case of anomalous diffusion near the accelerator mode of period five $\left(K=K_{c}=6.476939\right)$ and a case of normal diffusion $(K=6.0)$. The power-like law Eq. 4.9 is confirmed. In Fig. 6 we plot $\mu_{m}$ as a function of $m$. Two straight lines correspond to anomalous (solid squares) and normal (triangles) diffusion. The linear dependence of $\mu_{m}$ on $m$

$$
\mu_{m}=\mu_{1} \times m
$$

is a signature of the self-similarity. The values of $\mu_{1}$ are respectively $\mu_{1}=1$ for normal diffusion while for anomalous diffusion the value of $\mu_{1}$ is approximately the slope of the solid line, given by the theoretical anomalous exponent from Eq. 1.4. Determination of the higher moments gives much larger numerical errors. 


\section{RENORMALIZATION FOR THE EXIT TIME DISTRIBUTION}

Characteristic times have already been used by several authors to describe chaotic motion in relation to local structures in phase space $7^{7,26-29,11,12}$. In this section we use a new method developed for the web map and standard map ${ }^{22,23}$. In order to further investigate the sticking properties of the orbits near the border of the self-similar chains of islands we carried out detailed computer calculations of the distribution $\psi(t)$ of times it takes an orbit with initial coordinates placed close to a BIC to reach the chaotic sea.

To define $\psi(t)$ let us consider a small domain $\Delta \Omega$ in the phase space of a system and let $\psi(t ; \Delta \Omega)$ be the corresponding probability density to escape from $\Delta \Omega$ in a time interval $d t$. Then

$$
P_{e}(t ; \Delta \Omega)=\int_{0}^{t} \psi(\tau ; \Delta \Omega) d \tau
$$

is the probability for particles to leave the domain $\Delta \Omega$ during the time interval $t$. The survival probability is

$$
\Psi(t ; \delta \Omega)=1-P_{e}(t ; \Delta \Omega)=1-\int_{0}^{t} \psi(\tau ; \Delta \Omega) d \tau=\int_{t}^{\infty} \psi(\tau ; \Delta \Omega) d \tau
$$

where we used Eq. 5.1 and the condition of normalization

$$
P_{e}(t \rightarrow \infty ; \Delta \Omega)=1
$$

It is important to note that the functions $\psi, \Psi$ and $P_{e}$ are locally defined for a small domain $\Delta \Omega$ and can depend on the shape and location of this domain. If, for example, $\Delta \Omega$ is taken in a domain $\Delta \Omega_{s}$ of a singular zone around an island, then the probability $P_{e}$ to exit from the domain should be similarly dependent on $\mathrm{t}$ and should satisfy the self-similarity condition.

In order to formulate this property, we examine different self-similar domains $\Delta \Omega_{s}$, in the BIC of the corresponding generation order $k=0,1, \ldots .$. This means that they obey the law 
of self-similarity of the areas of Eq. 4.1. Fig. 7 shows the exit time distribution functions for the generations $0,1,2$ of the BIC. These distributions are computed with the use of the numerical method described in section III. Orbits with initial conditions which belong to the internal part of a resonance island of higher generation simply never escape, and thus do not contribute to the escape time statistics. They do however contribute to computing effort, and this is the reason for the careful choice of the initial domain B.

In the logarithmic scale there is a shift between the distributions of two successive selfsimilar generations. This shift is observable in Fig 7, which shows the escape time distributions at short times so that the third generation can also be displayed. The value of this shift is of the order of $\lambda_{T}=10.54$ (shift of 1 in $\log$ plot) which is the typical value of the temporal scaling parameter. It is apparent from Fig. 8 that the distribution of exit times follows a power-law with an exponent of the order of -3.5 , the slope of the line shown for comparison. (The normalizations of the distributions differ between Fig. 7 and Fig. 8, since the data sets are not equal.) Note that in short time computations the slope is about -1.6 but it changes drastically at large times. This result shows that the effect of long time memory which induces anomalous transport is indeed obtained only in large time computations, and is essentially due to the visits of the orbit to higher order generation islands of the BIC. Previous short time computations ${ }^{12,13}$ have found a smaller slope of about -1.6 and we find the same, but this value changes significantly at large times as can be seen in Fig. 8 .

Let us now investigate the distribution of the Poincaré cycles and compare it to the escape time distribution. Consider a small domain $\Delta \Gamma$ in the phase space of the system and take an initial condition $\left(x_{0}, p_{0}\right)$ belonging to this domain. Poincaré recurrence is the phenomenon of the trajectory passing through the domain $\Delta \Gamma$ infinitely many times. For 
the numerical computation of the probability distribution function of the Poincare cycles $P_{r}(t)$, we started with $4 \times 10^{4}$ initial conditions in a square box in the stochastic sea and found the return time distribution using $10^{7}$ iterations of the map. Fig. 9 shows $P_{r}(t)$ which has a Poisson distribution for short times but has a long time tail. This tail follows a power law with an exponent of the order of -3.5 which is compatible with the power law tail of the escape time distribution described above. Let us emphasize that at large times, the long time memory effects are mainly due to the visits of the orbits to the island generations of higher order and thus affect similarly all the characteristic times of the system.

\section{CONCLUSIONS}

The fine structure of the phase space of Hamiltonian systems is the primary origin of anomalous transport. Long-range correlation effects occur from visits of orbits to boundary layers in the vicinity of islands. This phenomenon is generally refered to as the stickiness of the islands. We have shown that for small changes of the stochasticity parameter strong topological changes can occur in the phase portrait of the system. As a concrete example we considered in this paper kinetic properties of the standard map near the threshold of the accelerator mode of period 5 and found a value of $\mathrm{K}$ producing an exact self-similar chain of islands associated with this accelerator mode. Spatio-temporal properties of this island chain have been numerically determined. We have shown that the kinetics is anomalous near the accelerator mode and corresponds to a superdiffusion process with a characteristic exponent related to the spatio-temporal scaling parameters of the island chain. We also found that the asymptotic behavior of the probability distribution of escape times from 
boundary domains of the self-similar chain of islands follows a power law, and that these escape times are renormalized according to the generation of the island considered. Moreover it was shown that the exponent of the power law which rules the escape time distribution is related to the transport exponent $\mu$ of Eq. 1.2.

Finally, the results of this paper emphasize the link between the self-similarity of the phase space and hence its topological properties and the kinetic properties of the orbits which tend to be anomalous because of long lasting memory effects introduced by this self-similarity. It is clear that these phenomena are encountered only for specific values of the stochasticity parameter for which there exist self-similar island chains. Close to these parameter values there exist values for which the self-similar chain has collapsed and normal diffusion pertains. Undoubtedly the set of parameter values giving rise to particular types of island chains is fractal, making the transport a very complex function of the map parameter. Let us conclude by remarking that we have investigated local transport properties of the system. These long time memory effects do not affect the global transport properties except at very large times.

\section{ACKNOWLEDGMENTS}

S. Benkadda and S. Kassibrakis wishes to thank princeton Plasma Physics laboratory for hospitality during their visit. R. White was supported by the U.S. Department of Energy under contract number DE-AC02-76-CHO3073. G. Zaslavsky was supported by the U. S. Navy Grant N00014-96-1-0055 and by the U.S. Department of Energy grant DE-FG0292ER54184. The authors would like to express their gratitude to M. Edelman for the help in providing computations. 


\section{REFERENCES}

1. B. V. Chirikov, Phys. Reports 52, 264 (1979).

2. A. B. Rechester and R. B. White, Phys. Rev. A 23, 2644 (1981).

3. A. B. Rechester, M. N. Rosenbluth, and R. B. White, Phys. Rev. Lett. 42, 1247 (1979).

4. A. J. Lichtenberg and M. A. Lieberman, Regular and Stochastic Motion, p. 293, Springer-Verlag, N. Y., 1983.

5. J. D. Meiss, J. R. Cary, C. Grebogi, J. D. Crawford, A. N. Kaufman, and H. D. I. Abarbanel, Physica D 6, 375 (1983).

6. I. Dana, N. Murray, and I. Percival, Phys. Rev. Lett. 62, 233 (1989).

7. C. C. F. Karney, Physica D 8, 360 (1983).

8. V. V. Beloshapkin and G. M. Zaslavsky, Phys. Lett. A 97, 121 (1983).

9. R. Ishizaki, T. Horita, T. Kobayashi, and T. Mori, Prog. Theor. Phys. 85, 1013 (1991).

10. R. Ishizaki, H. Hata, and T. Horita, Prog. Theor. Phys. 84, 179 (1990).

11. S. Benkadda, Y. Elskens, B. Ragot, and J. T. Mendonça, Phys. Rev. Lett. 7, 2859 (1994).

12. G. Zumofen and J. Klafter, Europhys. Letters 25, 565 (1994).

13. J. Klafter, G. Zumofen, and M. F. Shlesinger, in Lévy flights and Related Topics in Physics, p. 196, edited by M. F. Shlesinger, G. M. Zaslavsky, and U. Frisch, Springer, 1995. 
14. R. B. White, J. M. Rax, Y. Wu, M. N. Bussac, and L. Zuppiroli, in Transport, Chaos, and Plasma Physics, p153, edited by S. Benkadda, F. Doveil, and Y. Elskens, World Scientific, Singapore, 1994.

15. C. C. F. Karney, A. B. Rechester, and R. B. White, Physica D 4, 425 (1982).

16. E. W. Montroll and M. F. Shlesinger, in Studies in Statistical Mechanics, vol II, p. 1, edited by J. Lebowitz and E. W. Montroll, North-Holland, Amsterdam, 1984.

17. M. Shlesinger, G. Zaslavsky, and J. Klafter, Nature 363, 31 (1993).

18. B. A. Petrovichev, A. V. Rogalsky, R. Z. Sagdeev, and G. M. Zaslavsky, Phys. Lett. A 150, $3901(1990)$.

19. G. M. Zaslavsky, D. Stevens, and H. Weitzner, Phys. Rev. E 48, 1683 (1993).

20. G. M. Zaslavsky, Chaos 4, 25 (1994).

21. G. M. Zaslavsky, Physica D 76, 110 (1994).

22. G. M. Zaslavsky and B. A. Niyazov, Phys. Reports to appear.

23. G. M. Zaslavsky, M. Edelman, and B. A. Niyazov, Chaos to appear.

24. R. B. White, J. M. Rax, and Y. Wu, Transport in a stochastic magnetic field, in Statistical Description of Transport in Plasma, Astro-, and Nuclear Physics, edited by J. Misguich and G. Pelletier, Nova Science, New York, 1993.

25. R. B. White, Theory of Tokamak Plasmas, p. 19, North Holland, 1989.

26. B. V. Chirikov and D. Shepeliansky, Physica D 13, 394 (1984). 
27. B. V. Chirikov, Chaos, Solitons and Fractals 1, 79 (1991).

28. W. Easton, J. D. Meiss, and S. Carver, Chaos 3, 153 (1993).

29. G. Zaslavsky and M. Tipett, Phys. Rev. Lett. 67, 3251 (1991). 


\section{FIGURES}
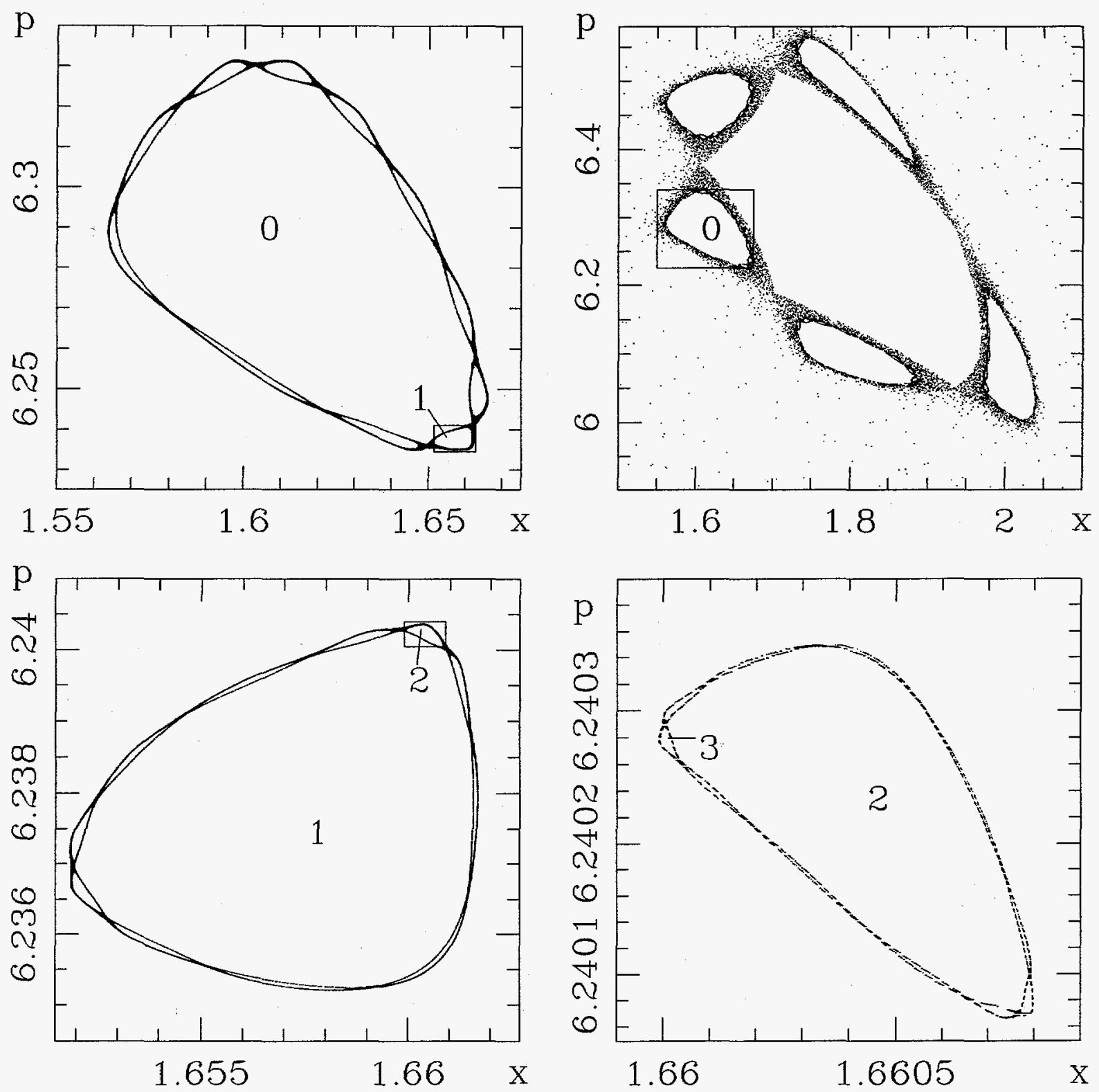

Fig. 1. Poincaré plot- 4 generations of self-similar islands. 

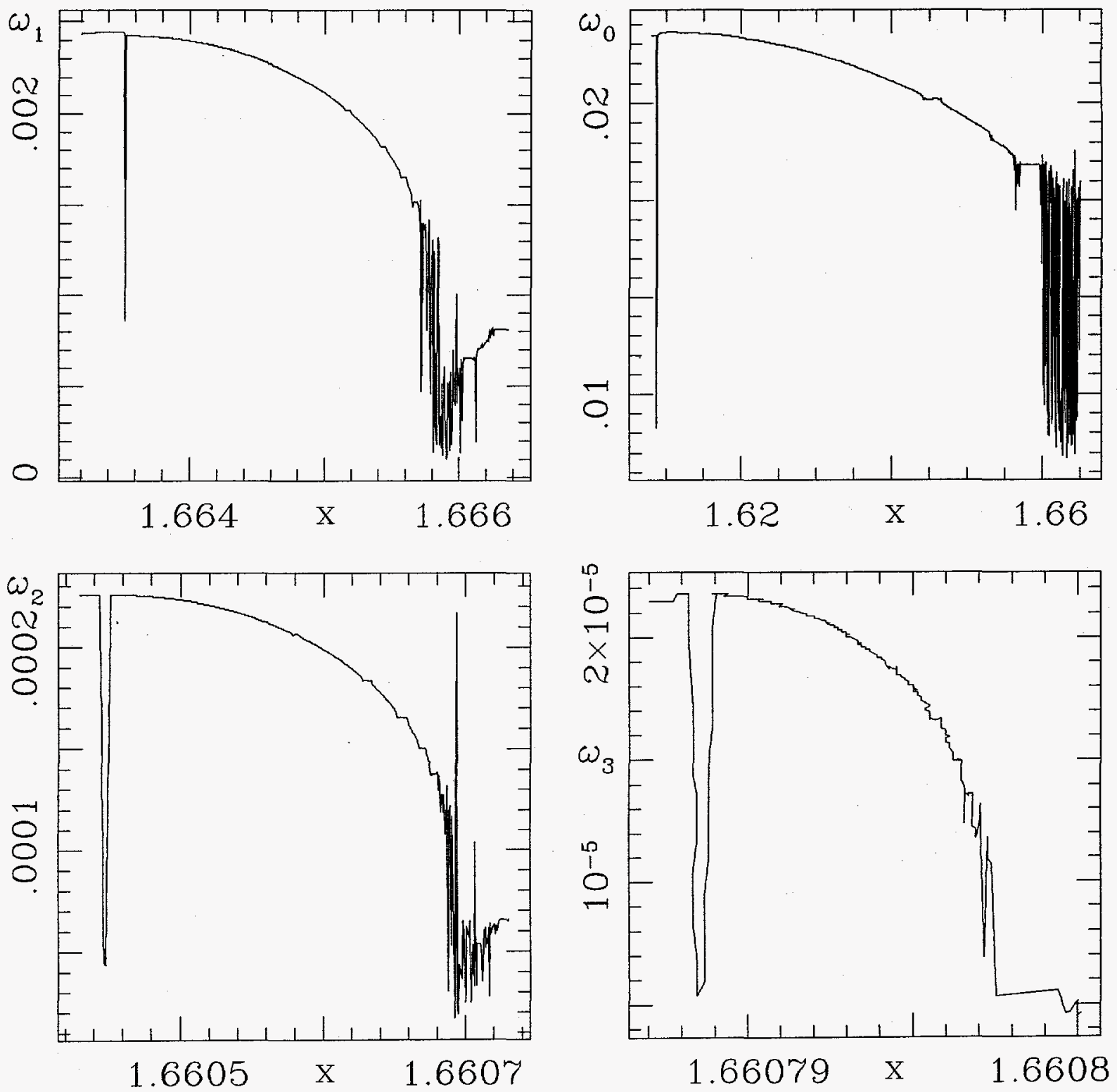

Fig. 2. $\omega$ profiles -4 generations of self-similar islands. 


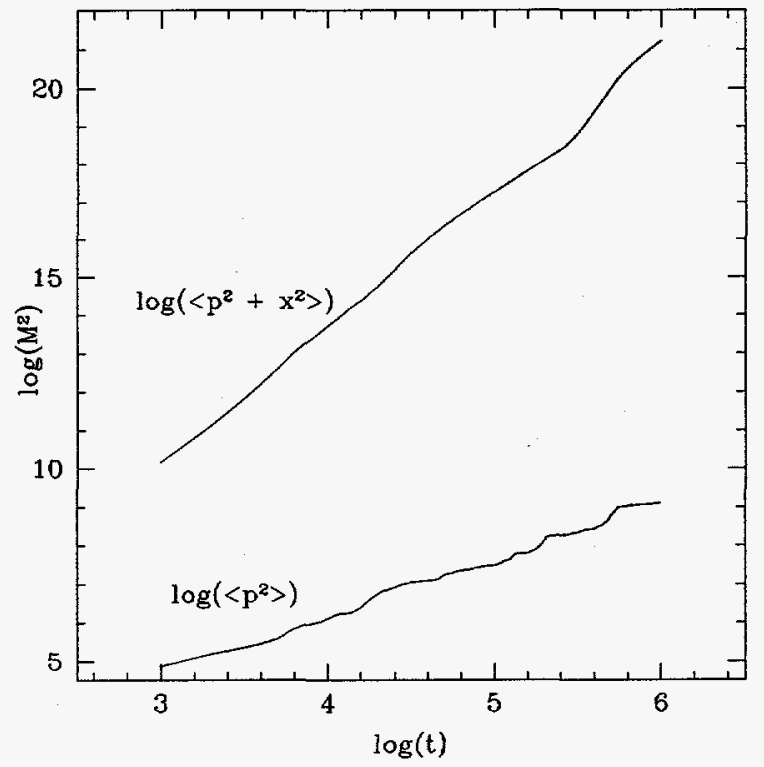

Fig. 3. Second order moments of $\left\langle p^{2}\right\rangle,\left\langle p^{2}+x^{2}\right\rangle$ on the cylinder and the plane.

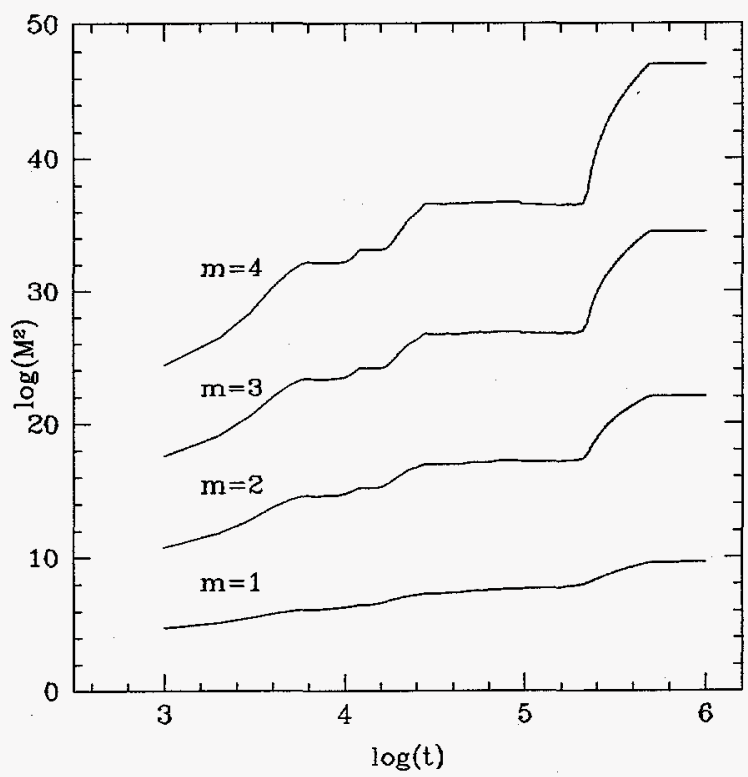

Fig. 4. Higher order moments, anomalous diffusion. 


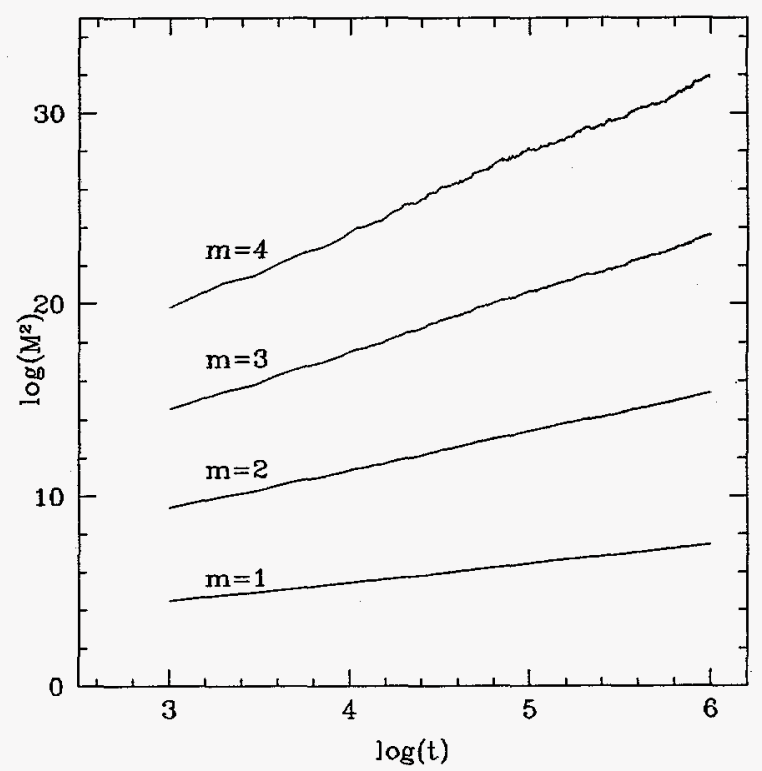

Fig. 5. Higher order moments, normal diffusion.

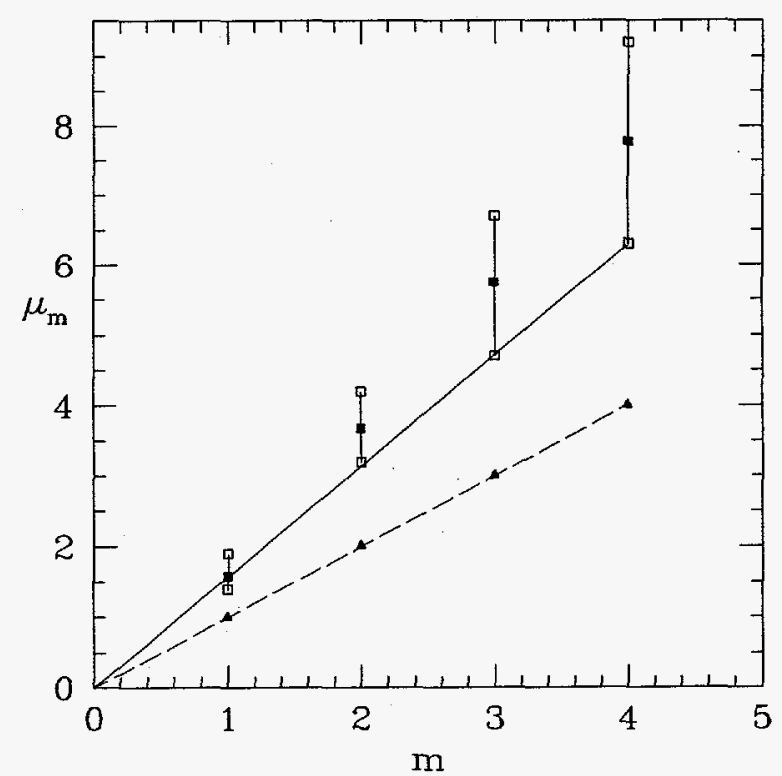

Fig. 6. $\mu_{m}$ as a function of $\mathrm{m}$ for normal and anomalous diffusion. 


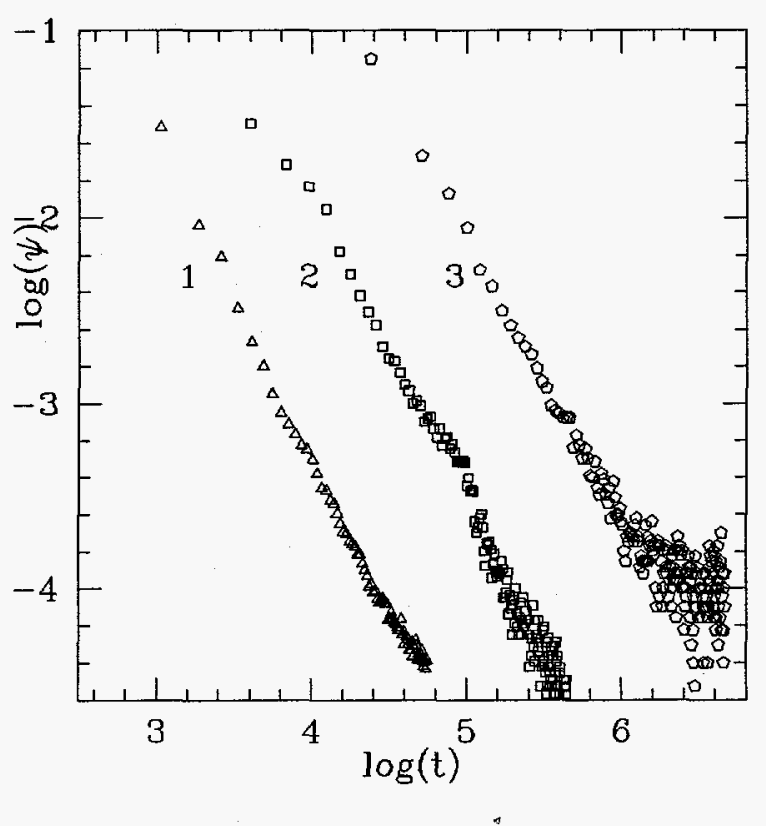

Fig. 7. Escape time distributions at short times, generations $1,2,3$.

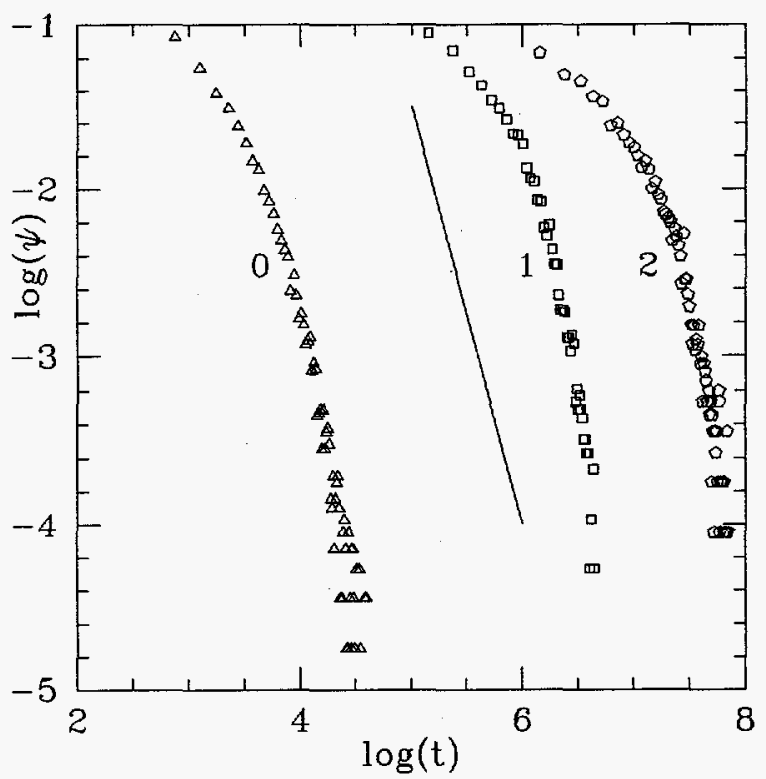

Fig. 8. Escape time distributions at large times, generations 0,1,2. 


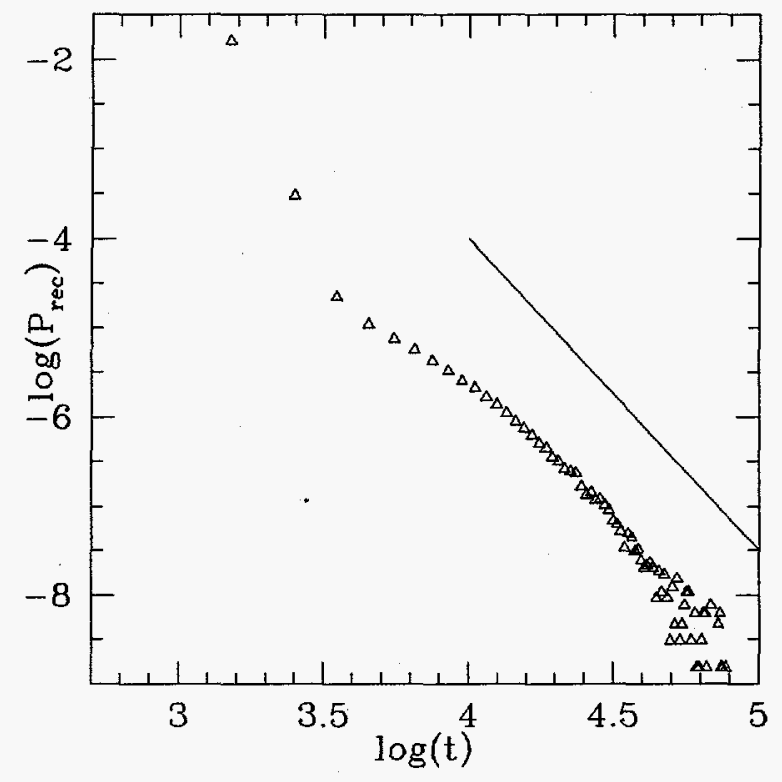

Fig. 9. Recurrence time distribution, stochastic sea initial conditions. 
Figure Captions

Fig. 1 Poincaré plot- 4 generations of self-similar islands.

Fig. $2 \omega$ profiles- 4 generations of self-similar islands.

Fig. 3 Second order moments of $\left\langle p^{2}\right\rangle,\left\langle p^{2}+x^{2}\right\rangle$ on the cylinder and the plane.

Fig. 4 Higher order moments, anomalous diffusion.

Fig. 5 Higher order moments, normal diffusion.

Fig. $6 \mu_{m}$ as a function of $\mathrm{m}$ for normal and anomalous diffusion.

Fig. 7 Escape time distributions at short times, generations 1,2,3.

Fig. 8 Escape time distributions at large times, generations $0,1,2$.

Fig. 9 Recurrence time distribution, stochastic sea initial conditions. 